\title{
An XMRV Derived Retroviral Vector as a Tool for Gene Transfer
}

Daniel Cervantes-Garcia ${ }^{1,2}$, Augusto Rojas-Martinez ${ }^{2}$ and David Camerini ${ }^{1 *}$

\begin{abstract}
Background: Retroviral vectors are widely used tools for gene delivery and gene therapy. They are useful for gene expression studies and genetic manipulation in vitro and in vivo. Many retroviral vectors are derived from the mouse gammaretrovirus, murine leukemia virus (MLV). These vectors have been widely used in gene therapy clinical trials. XMRV, initially found in prostate cancer tissue, was the first human gammaretrovirus described.

Findings: We developed a new retroviral vector based on XMRV called pXC. It was developed for gene transfer to human cells and is produced by transient cotransfection of LNCaP cells with PXC and XMRV-packaging plasmids.

Conclusions: We demonstrated that PXC mediates expression of inserted transgenes in cell lines. This new vector will be a useful tool for gene transfer in human and non-human cell lines, including gene therapy studies.
\end{abstract}

Keywords: XMRV, retroviral vector, transduction

\section{Findings}

Retroviral vectors offer a highly efficient method of stable gene transfer in mammalian cells due to their ability to integrate into the host genome [1,2]. Moreover, the common genetic architecture of most retroviruses allows the development of similar retroviral vectors with different potentials for cell entry via virusspecific receptors and different capabilities for gene expression mediated by diverse retroviral promoters [3].

Current retroviral vectors used for gene transfer are replication defective. Trans-expression of retroviral structural proteins from non vector-homologous plasmids avoids the production of replication competent retrovirus (RCR) [4,5]. Many retroviral vectors are derived from murine leukemia virus (MLV) in both, ecotropic and amphotropic versions [6,7]. Lentiviral vectors based on HIV may offer advantages because of their lower insertion frequency in crucial loci involved in cell growth regulation and their ability to transduce non-dividing cells $[8,9]$. Nevertheless, MLV-derived retroviral vectors have been used extensively, including in more than 300 gene therapy clinical trials [10]. In addition, retroviral vectors derived from avian sarcoma

\footnotetext{
* Correspondence: david.camerini@uci.edu

'Division of Infectious Diseases, Center for Virus Research and Institute for Immunology, University of California, Irvine, California, 92697-4068, USA Full list of author information is available at the end of the article
}

leukosis virus (ASLV; [11]), spleen focus-forming virus (SFFV; [12]), and Mason-Pfizer monkey virus (MPMV; [13]), have been developed among others.

In 2006, the xenotropic murine-leukemia-virus related gammaretrovirus (XMRV) was discovered in a subset of human prostate cancer ( $\mathrm{PCa}$ ) tissue samples [14]. Subsequently, additional studies demonstrated that XMRV uses the XPR1 receptor to initiate infection, and that the virus is sensitive to IFN- $\beta$ and RNase-L, a final effector of the IFN- $\beta$ mediated antiviral response [15]. Since XMRV shows the basic structure of gammaretroviruses, we developed a panel of packaging plasmids and retroviral vectors derived from XMRV. Here we demonstrate their potential use as gene transfer vectors for in vitro assays.

Initially, we constructed a plasmid to evaluate the promoter activity of the U3 region of the XMRV LTR derived from $22 \mathrm{Rv} 1$ cells. A fragment of 554 base pairs was isolated by PCR with oligonucleotide primers XMRV-U3-f and XMRV-U3-r (Table 1), and inserted into the plasmid vector, pCR2.1-Topo (Invitrogen). Digestion with the enzymes $S a c$-I and Xho-I released a fragment of 632 base pairs, which was inserted into the plasmid vector, pGL3-Basic (Promega). The pGL3XMRV-U3-luc expression plasmid was used to transfect Hep G2, HEK-293, SiHa, 22Rv1, and PC-3 cell lines. Luciferase expression was assayed 72 hours later. Similar
C Biomed Central

(c) 2011 Cervantes-Garcia et al; licensee BioMed Central Ltd. This is an Open Access article distributed under the terms of the Creative Commons Attribution License (http://creativecommons.org/licenses/by/2.0), which permits unrestricted use, distribution, and reproduction in any medium, provided the original work is properly cited. 
Table 1 Oligonucleotide primers used in this study.

\begin{tabular}{|c|c|c|}
\hline Oligonucleotide & $5^{\prime}$ modification & Sequence \\
\hline \multicolumn{3}{|c|}{ Luciferase expression plasmid } \\
\hline XMRV-U3-f & - & GCCCTGGTTCTGACCCAACAGTAT \\
\hline XMRV-U3-r & - & AAAGGCTTTATTGGGAACACGGGT \\
\hline \multicolumn{3}{|l|}{ Vector plasmid } \\
\hline CMV-IEE-f & Sacl & GAGCTCCGCGTTACATAACTTACGG \\
\hline CMV-IEE-r & Mfel & СAATTGCAAAACAAACTCCCATTGACG \\
\hline XMRV-LTR5-f & Mfel & $\underline{\text { CAATTGTGAAAGACCCCACCATAAGG }}$ \\
\hline XMRV- $\triangle$ gag-r & Avrll & 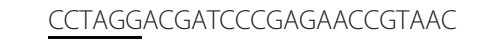 \\
\hline CMV-IEP-f & Avrll & CCTAGGGTTGACATTGATTATTGAC \\
\hline CMV-IEP-r & Xhol & CTCGAGGTCTGCTTATATAGACC \\
\hline XMRV-PPT-LTR3-f & Xbal & TCTAGAATTCGGTAGTGCAGGCCCTGG \\
\hline XMRV-PPT-LTR3-r & Spel & ACTAGTAATGAAAGACCCCCGAGCTGGG \\
\hline \multicolumn{3}{|c|}{ Packaging plasmid gag-pol } \\
\hline XMRV-gagpol-f & Nhel & GCTAGCATCATGGGACAGACCGTAACTAC \\
\hline XMRV-gagpol-r & Notl & GCGGCCGCTTAGGGAAAGTGTCTGTCATCGT \\
\hline \multicolumn{3}{|c|}{ Packaging plasmid env } \\
\hline XMRV-env-f & Kpnl & GGTACCCATGGAAATGCCAGCGTTCTCAA \\
\hline XMRV-env-r & Notl & GCGGCCGCGCTAGCGTGCTAAGCCTTAT \\
\hline
\end{tabular}

levels of transcription were observed in all five cell lines tested and little or no cell type specificity of the XMRV promoter was found. Moreover, the level of luciferase expression mediated by the XMRV promoter was similar to that mediated by the SV40 virus early region promoter in each cell line (Figure 1).

An XMRV-derived retroviral gene transfer vector, pXC, was constructed in the pBluescript II KS plasmid. Genomic DNA from 22Rv1 cells was used as template for XMRV sequence isolation by PCR amplification. The CMV immediate early enhancer (CMV-IEE) was positioned 5 ' to the XMRV LTR and XMRV RNA-packaging

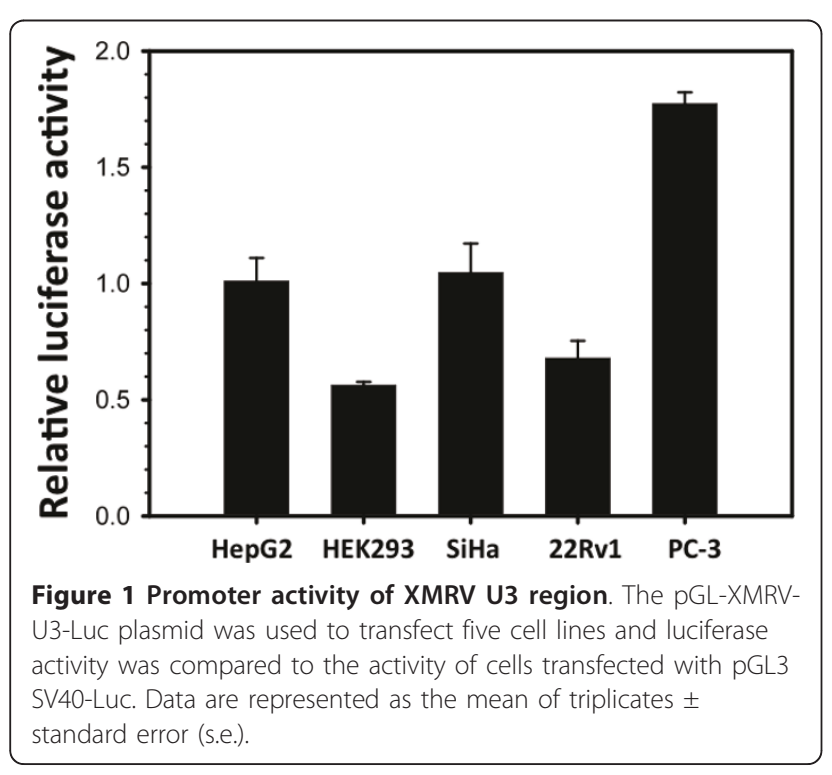

signal, followed by the CMV immediate early promoter (CMV-IEP) driving a reporter gene (GFP or luciferase), the XMRV polypurine tract, and a 3' XMRV LTR (Figure 2). GFP or luciferase genes were inserted in the $\mathrm{Xho}-\mathrm{I} / \mathrm{Sal}$-I and $\mathrm{Xba}-\mathrm{I}$ sites. XMRV vector packaging plasmids pcDNA3.1/Hygro-XMRVgagpol and pcDNA3.1/Zeo-XMRVenv were also created by amplification of the gag-pol and env genes, respectively. The templates for isolation of XMRV structural genes were

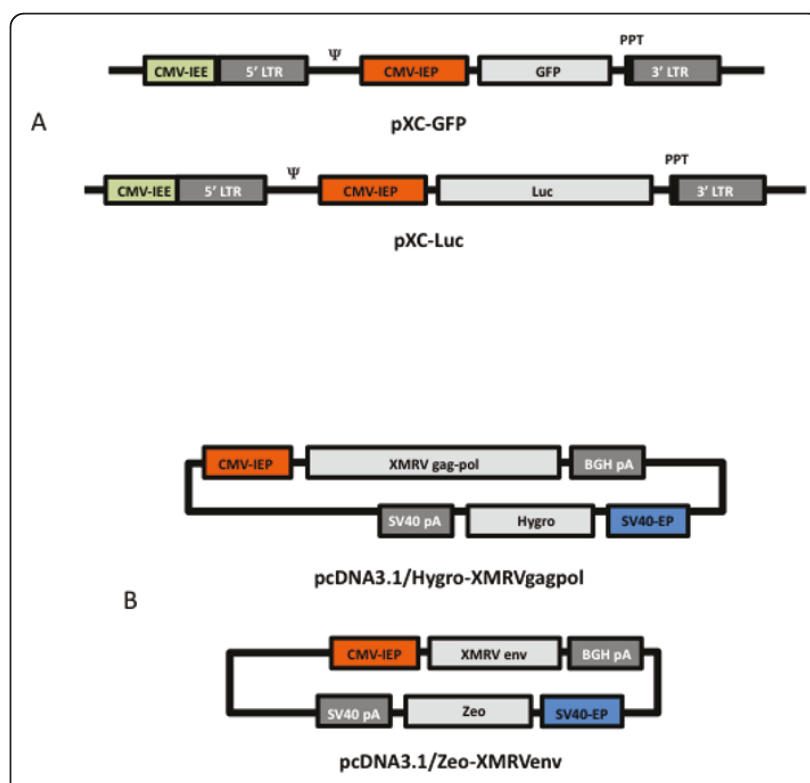

Figure 2 Vectors and packaging plasmids. Schematic maps of $(A)$ XMRV-derived vectors, and (B) XMRV vector packaging plasmids. 
the VP62 clone [14] for the gag-pol gene, and genomic DNA from the 22Rv1 line for the env gene. Primers used for these steps are described in Table 1.

XMRV structural proteins were expressed in LNCaP cells, since these cells satisfactorily support XMRV protein expression and replication [16,17]. LNCaP cells were transfected with the packaging plasmids $(50 \mu \mathrm{g}$ each) using Lipofectamine and XMRV Gag and Env protein expression was detected by SDS-PAGE followed by western blotting (Figure 3). Ninety-six hours post-transfection, cells were harvested and proteins were extracted with lysis buffer (1\% Triton X-100, $10 \mathrm{mM}$ Tris- $\mathrm{HCl} \mathrm{pH}$ 7.5, $50 \mathrm{mM} \mathrm{KCl,} 2 \mathrm{mM} \mathrm{MgCl}_{2}, 0.5 \mathrm{mM}$ PMSF, 0.02 $\mathrm{mM} \beta$-mercaptoethanol, and protease inhibitor cocktail). The XMRV capsid protein (p30) and surface glycoprotein (gp70) were detected using rat monoclonal anti-p30 antibody (kindly provided by Drs. Sandra and Francis Ruscetti), goat anti-gp70 serum (kindly provided by Dr. John Elder), goat anti-rat IgG conjugated to horseradish peroxidase (HRP; Invitrogen), and chicken anti-goat IgG conjugated to HRP (Santa Cruz Biotech) respectively. HRP activity was detected by chemiluminescence using a commercial kit (Pierce).

Lipofectamine mediated triple transfection of LNCaP cells with the XMRV-derived vector $\mathrm{pXC}$-Luc or $\mathrm{pXC}$ GFP and two XMRV vector packaging plasmids, pcDNA3.1/Hygro-XMRVgagpol and pcDNA3.1/Zeo$\mathrm{XMRV}$ nv, were used to produce XMRV-derived retroviral vectors in the culture media. Cell free supernatants were filtered 72 hours after transfection to produce XMRV vector stocks. Vector stocks were centrifuged onto LNCaP cells at 2500 RPM for 90 minutes at room temperature (spinfected) in the presence of $8 \mu \mathrm{g} / \mathrm{mL}$ polybrene. Seventy-two hours later, luciferase expression in the spinfected LNCaP cells was assayed with a commercial assay kit (Dual-Glo Luciferase Assay System, Promega). The level of luciferase expression achieved in LNCaP cells transduced with pXC-Luc was more than 320-fold greater than the background expression following transduction with pXC-GFP (Figure 4). Luciferase expression mediated by $\mathrm{pXC}$-Luc, however, was lower
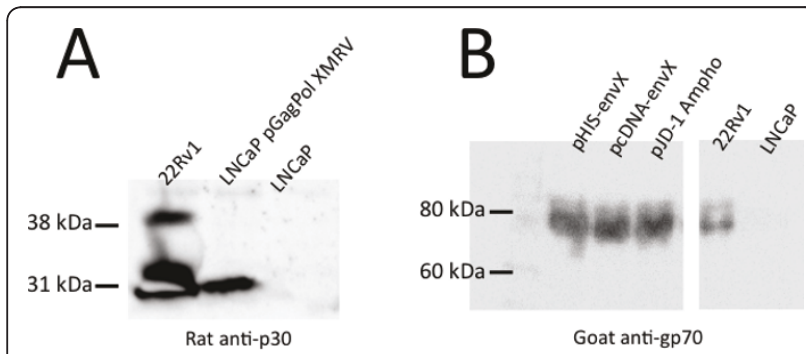

Figure 3 XMRV structural proteins. Western blots were performed for detection of the p30 capsid subunit of Gag and the gp70 surface glycoprotein subunit of Env in LNCaP cells.

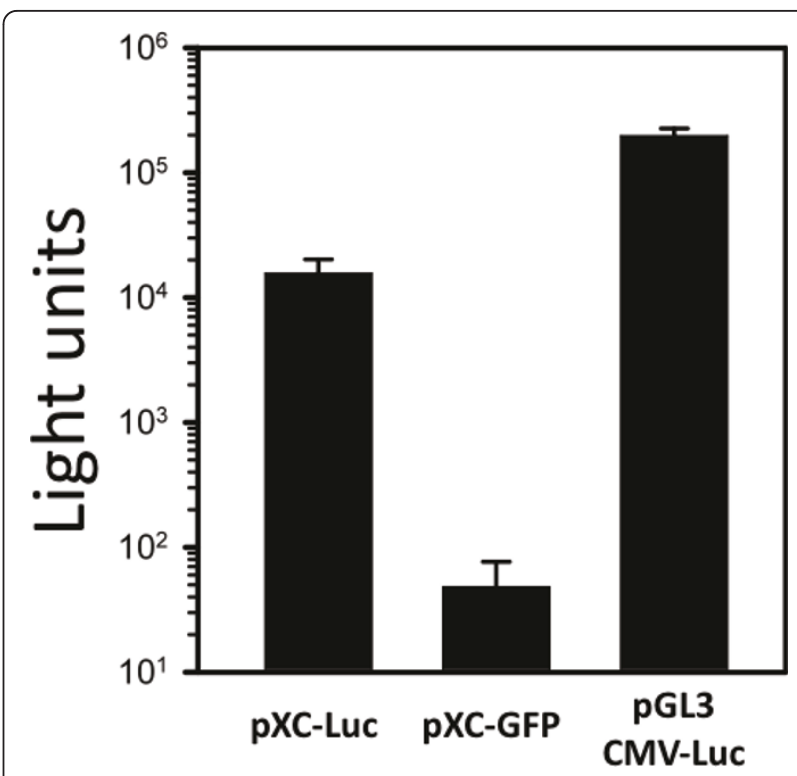

Figure 4 Gene expression mediated by XMRV-derived vector in LNCaP cells. Gene transfer vector XC-Luc or XC-GFP were used to transduce LNCaP cells. Luciferase activity was measured 72 hours after transduction. As a positive control, LNCaP cells were transfected with the plasmid pGL3 CMV-Luc using Lipofectamine. Data are represented as the mean of triplicates \pm standard error ( $\mathrm{s}$. e.).

than expression in LNCaP cells transfected with pGL3 CMV-Luc.

293T and HeLa cells were also transduced with pXCLuc and these cells were analyzed for luciferase expression 72 hours later along with LNCaP cells (Figure 5). Consistent with previous reports, HeLa and 293T cells were transduced with $\mathrm{pXC}$, but luciferase expression was lower than in LNCaP cells.

\section{Conclusions}

Many retroviral vectors for gene transfer are derived from murine gamma-retroviruses. Nevertheless, to our knowledge, the pXC vector and its associated cell packaging system described here constitute the first retroviral vector system for gene transfer based on a likely human gammaretrovirus, with particular tropism for human cells and tissues. Our results suggest that the pXC vector is a useful tool for gene transfer in human cells and it is possible that this vector will contribute to elucidation of the interactions of XMRV and its human host. In addition, this new XMRV-derived retroviral vector has the potential to be used for stable transfection of human cells and for preclinical studies of gene therapy.

\section{Acknowledgements}

We thank UC-MEXUS CONACYT for support of this study with grant number CN08-229. We also thank Drs. Sandra and Francis Ruscetti, and Dr. John Elder, for providing primary antibodies. 


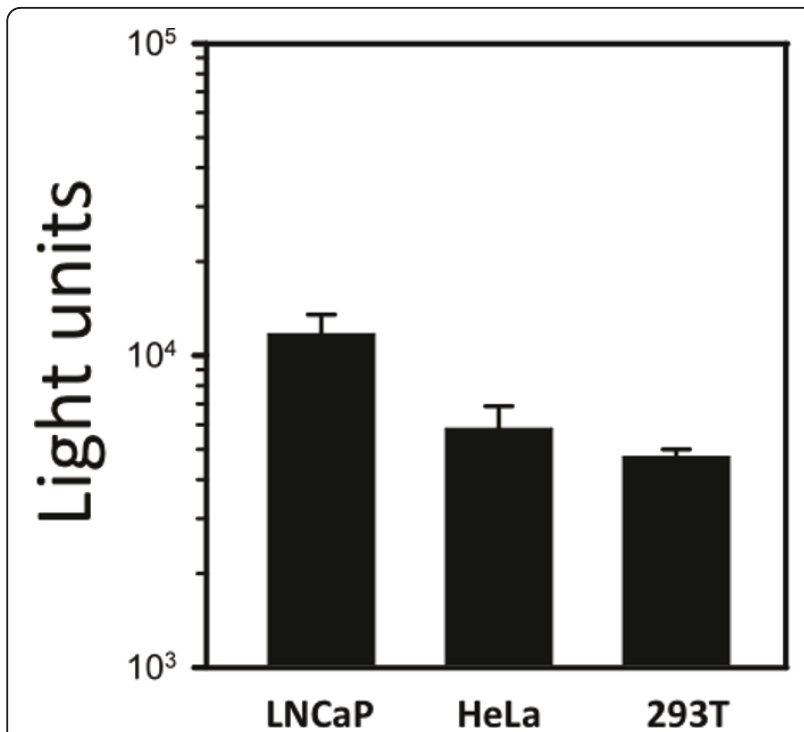

Figure 5 Transduction of different cell lines with the XMRVderived vector. Luciferase activity in LNCaP, HeLa, and 293T cell lines 72 hours after transduction with the XMRV-derived vector, pXC-luc. Data are represented as mean of triplicates \pm standard error (s.e.).

\section{Author details}

'Division of Infectious Diseases, Center for Virus Research and Institute for Immunology, University of California, Irvine, California, 92697-4068, USA. ${ }^{2}$ Department of Biochemistry and Molecular Medicine in the School of Medicine, Centro de Investigacion y Desarrollo en Ciencias de la Salud, Unidad de Terapia Genica y Celular, Universidad Autonoma de Nuevo Leon, Monterrey, Nuevo Leon, Mexico.

\section{Authors' contributions}

DCG participated in gene isolation, plasmid construction, transduction assays, and writing this manuscript. ARM conceived of the study, helped interpret the data and write the manuscript. DC aided in the design and coordination, the interpretation of the data and helped to draft the manuscript. All authors read and approved the final manuscript.

\section{Competing interests}

The authors declare that they have no competing interests.

Received: 20 May 2011 Accepted: 8 June 2011 Published: 8 June 2011

\section{References}

1. Page KA, Landau NR, Littman DR: Construction and use of a human immunodeficiency virus vector for analysis of virus infectivity. J Virol 1990, 64:5270-5276.

2. Thomas CE, Ehrhardt A, Kay MA: Progress and problems with the use of viral vectors for gene therapy. Nat Rev Genet 2003, 4:346-358.

3. Hu WS, Pathak VK: Design of retroviral vectors and helper cells for gene therapy. Pharmacol Rev 2000, 52:493-511.

4. Kim S, Park EJ, Yu SS, Kim S: Development of enzyme-linked immunosorbent assay for detecting antibodies to replication-competent murine leukemia virus. J Virol Methods 2004, 118:1-7.

5. Sinn PL, Sauter SL, McCray PB Jr: Gene therapy progress and prospects: development of improved lentiviral and retroviral vectors-design, biosafety, and production. Gene Ther 2005, 12:1089-1098.

6. Xu L, O'Malley T, Sands MS, Wang B, Meyerrose T, Haskins ME, Ponder KP: In vivo transduction of hematopoietic stem cells after neonatal intravenous injection of an amphotropic retroviral vector in mice. Mol Ther 2004, 10:37-44.
7. Koch P, Siemen H, Biegler A, Itskovitz-Eldor J, Brüstle O: Transduction of human embryonic stem cells by ecotropic retroviral vectors. Nucleic Acids Res 2006, 34:e120

8. D'Costa J, Mansfield SG, Humeau LM: Lentiviral vectors in clinical trials: Current status. Curr Opin Mol Ther 2009, 11:554-564.

9. Pluta K, Kacprzak MM: Use of HIV as a gene transfer vector. Acta Biochim Pol 2009, 56:531-595.

10. Gene Therapy Clinical Trials Worldwide. [http://www.wiley.com//legacy/ wileychi/genmed/clinical/]

11. Torne-Celer C, Moreau K, Faure C, Verdier G, Ronfort C: An improved selfdeleting retroviral vector derived from avian leukemia and sarcoma virus. Arch Virol 2008, 153:2233-2243.

12. Marusich El, Parveen Z, Strayer D, Mukhtar M, Dornburg RC, Pomerantz RJ: Spleen necrosis virus-based vector delivery of anti-HIV-1 genes potently protects human hematopoietic cells from HIV-1 infection. Virology 2005, 332:258-271.

13. Guesdon FM, Greatorex J, Rhee SR, Fisher R, Hunter E, Lever AM: Sequences in the $5^{\prime}$ leader of Mason-Pfizer monkey virus which affect viral particle production and genomic RNA packaging: development of MPMV packaging cell lines. Virology 2001, 288:81-88.

14. Urisman A, Molinaro RJ, Fischer N, Plummer SJ, Casey G, Klein EA, Malathi K, Magi-Galluzzi C, Tubbs RR, Ganem D, Silverman RH, DeRisi JL: Identification of a novel Gammaretrovirus in prostate tumors of patients homozygous for R462Q RNASEL variant. PLOS Pathog 2006, 2(3):e25.

15. Dong B, Kim S, Hong S, Das Gupta J, Malathi K, Klein EA, Ganem D, Derisi JL, Chow SA, Silverman RH: An infectious retrovirus susceptible to an IFN antiviral pathway from human prostate tumors. Proc Natl Acad Sci USA 2007, 104:1655-1660.

16. Bhosle S, Suppiah S, Molinaro R, Liang Y, Arnold R, Diehl W, Makarova N, Blackwell J, Petros J, Liotta D, Hunter E, Ly H: Evaluation of cellular determinants required for in vitro xenotropic murine leukemia virusrelated virus entry into human prostate cancer and noncancerous cells. J Virol 2010, 84(13):6288-6296.

17. Rodriguez JJ, Goff SP: Xenotropic murine leukemia virus-related virus establishes an efficient spreading infection and exhibits enhanced transcriptional activity in prostate carcinoma cells. J Virol 2010, 84(5):2556-2562.

doi:10.1186/1743-422X-8-284

Cite this article as: Cervantes-Garcia et al:: An XMRV Derived Retroviral Vector as a Tool for Gene Transfer. Virology Journal 2011 8:284.

\section{Submit your next manuscript to BioMed Central and take full advantage of:}

- Convenient online submission

- Thorough peer review

- No space constraints or color figure charges

- Immediate publication on acceptance

- Inclusion in PubMed, CAS, Scopus and Google Scholar

- Research which is freely available for redistribution 